\section{PSICOLOGIA IBEROAMERICANA}

\section{Psicología lberoamericana}

ISSN: 1405-0943

psicología.iberoamericana@uia.mx

Universidad Iberoamericana, Ciudad de

México

México

Hernández Zamora, Zoila Edith; Hernández Loeza, Oralia Rocío; Rodríguez Viveros, Erika

El Grupo de Ayuda como Alternativa para Mejorar la Calidad de Vida del Adulto Mayor

Psicología Iberoamericana, vol. 18, núm. 2, julio-diciembre, 2010, pp. 47-55

Universidad Iberoamericana, Ciudad de México

Distrito Federal, México

Disponible en: http://www.redalyc.org/articulo.oa?id=133915921006

Cómo citar el artículo

Número completo

- Más información del artículo

- Página de la revista en redalyc.org

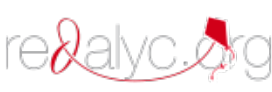

Sistema de Información Científica

Red de Revistas Científicas de América Latina, el Caribe, España y Portugal

Proyecto académico sin fines de lucro, desarrollado bajo la iniciativa de acceso abierto 


\title{
El Grupo de Ayuda como Alternativa para Mejorar la Calidad de Vida del Adulto Mayor
}

\author{
Assisting Group as an Alternative to Improve Quality of Life of Aged People
}

\author{
Zoila Edith Hernández Zamora \\ Oralia Rocío Hernández Loeza \\ Erika Rodríguez Viveros* \\ INSTITUTO dE INVESTIGACIONES PSICOLÓGICAS \\ Universidad Veracruzana, Xalapa, México
}

\section{RESUMEN}

Esta investigación, de corte cualitativo, reporta la creación de un grupo de ayuda para los adultos mayores como una alternativa para mejorar su calidad de vida. El grupo estuvo conformado por ocho mujeres mayores de 60 años pertenecientes al programa Oportunidades de un Centro de Salud de la ciudad de Banderilla, Veracruz, México. Todas del sexo femenino y con un rango de edad de 61 a 72 años. Se creó un espacio de escucha y de diálogo para las participantes, a fin de generar un grupo que, una vez finalizado el programa de intervención, fuera auto organizativo y de gestión. El desarrollo del programa tuvo tres fases: 1. poner en conocimiento de los miembros del grupo las bases teóricas y prácticas de la autoayuda, la metodología de las sesiones y las normas del grupo, 2. entrenar al grupo en la práctica metodológica del grupo de autoayuda, y 3. lograr que el grupo consiga su autonomía al llevar las sesiones sin el acompañamiento del coordinador o profesional. Las categorías que se trabajaron fueron: estado de salud en general, soporte social, autonomía y actividades físico recreativas. Los resultados revelaron un impacto en su calidad de vida en cuanto a los rubros señalados en la entrevista inicial. Con esto se concluye que un grupo de ayuda puede ser una alternativa para mejorar la calidad de vida de los adultos mayores.

Descriptores: Grupo de ayuda, mujeres mayores, salud, apoyo social, calidad de vida.

\section{ABSTRACT}

The present research, of qualitative type, reports the creation of an assisting group as an alternative to improve the quality of life of aged people. The group included eight women older than 60 (61-72), belonging to a governmental social program: Oportunidades, of a Helath Center in the city of Banderilla, Veracruz, Mexico. The program foresees the creation of an environment in which participants are able to be listened and to talk in order to form a group that, once the intervention program has ended, they can organize and manage in an autonomous way. The development of the program had three stages: 1 . To inform the group about the theoretical and practical basis of the self-help, the methodology of the sessions and the rules of the group, 2. to train the group for practicing the methodology of the self-help group and 3. to make the group autonomous in carrying out the sessions without the coordination of a professional. The categories included were: health state, social support, autonomy and recreational and physical activities. The results showed an impact on their life quality regarding the categories included in the initial interview. The results suggest that the creation of an assisting group may be an alternative to improve quality of life of aged people.

Key words: assisting group, aged women, health, social support, quality of life.

\footnotetext{
*Para correspondencia: Zoila Edith Hernández Zamora. Instituto de Investigaciones Psicológicas de la Universidad Veracruzana, Dr. Luis Castelazo Ayala s/n, Col. Industrial Ánimas, 91190, Xalapa, Ver., México, tel. (228) 8418900 ext. 13219, correo electrónico: zhernandez@uv.mx. Oralia Rocío Hernández Loeza. Facultad de Psicología de la Universidad Veracruzana, Manantiales de San Cristóbal s/n. Xalapa 2000, 91190. Xalapa, Ver., México, tel. (228) 8191555, correo electrónico: orla_her@hotmail.com. Erika Rodríguez Viveros. Facultad de Psicología de la Universidad Veracruzana, Manantiales de San Cristóbal s/n. Xalapa 2000, 91190. Xalapa, Ver., México, tel. (228) 8191555, correo electrónico: erika.rodriguezv@hotmail.com.
} 


\section{INTRODUCCIÓN}

La población de adultos mayores hoy en día es considerada como un tópico importante a tratar dentro de la investigación, ya que se tiene estimado que en un futuro, a nivel mundial, una de cada cuatro personas pertenezca a este grupo etario (González, 2000).

A principios del siglo XXI la esperanza de vida en México es de 72 años para los hombres y de 77 para las mujeres (INEGI, 2006) y en un futuro podría decirse que estas edades serán sobrepasadas. Esto se debe principalmente a los avances de la medicina, que hacen posible la prolongación de vida; sin embargo, es deber de la sociedad y sus miembros trabajar porque ésta sea vivida de manera satisfactoria y digna, cosa que es muy difícil para las personas mayores, ya que se trata de una población que está expuesta, en determinados sectores sociales y/o familiares, a que se le denigre de forma moral, económica, psicológica, física y/o socialmente (Carretero, Palacios \& Marchesi, 2000).

Una persona mayor es definida convencionalmente como toda aquella que tiene 60 o más años. Alrededor de esta edad pueden surgir, dependiendo mucho de las características de cada persona y de su historia de vida, cambios biopsicosociales entre los que se encuentran, según Carretero et al., 2000:

1. Esta etapa es considerada como la última de la vida, por lo que frecuentemente se relaciona con la muerte, cosa que disminuye la motivación y autoestima, afectando los proyectos de vida.

2. Dentro del ámbito laboral se puede considerar poco o nada productiva a esta población, por lo que les resulta muy difícil encontrar trabajo y como consecuencia se ve afectado su estado financiero, o bien, pueden encontrarse jubilados.

3. En la familia, en algunos casos son considerados como una carga, ya que tienen que mantenerlos y cuidar de ellos.

4. Algunos pueden sufrir cambios físicos, emocionales, sociales y biológicos por lo que, de no contar con un apoyo social satisfactorio, tales cambios influyen para la aparición de trastornos depresivos y otros trastornos emocionales.

En lo que respecta a este último punto, se ha comprobado que cuando los adultos mayores participan en re- des sociales de forma activa, logran sentirse mejor que aquellos que no participan y que la creación de vínculos con otras personas sirve de apoyo socioemocional en sus vidas. De manera que la fuente de apoyo que las personas mayores puedan recibir de la familia, del apoyo social informal (vecinos amigos) y del formal (servicios sociales, ayuntamientos, órganos estatales, entre otros) resulta esencial para una vejez feliz y exitosa (Triado \& Villar, 2006).

En relación a la importancia de los grupos en la vida de las personas, ésta se ve reflejada desde su nacimiento, ya que al nacer, crecer y desarrollarse siempre se está inmerso dentro de un grupo, como lo pueden ser: la familia, escuelas e inclusive dentro de alguna institución donde se esté laborando o en la que se compartan intereses. Además, dentro de una sociedad siempre van a existir los grupos, ya sean grandes o pequeños, que dirijan nuestras aspiraciones hacia metas concretas, como los familiares, de amigos, laborales, deportivos, religiosos, sociales, entre otros; lo cual pone de manifiesto que una buena parte de nuestras experiencias estarán relacionadas por una u otra causa, con los grupos.

Marín y Garrido (2003) mencionan que el mismo grupo como tal proporciona modos de percibir la realidad, como son los valores o estereotipos y/o cómo conducirse ante esa realidad, como las actitudes y las normas. Esto implica que gran parte de nuestros comportamientos se los debemos a la sociedad.

Por otro lado, el grupo también puede contribuir al logro de un cambio emocional para los participantes, es decir, puede servir como un apoyo para conservar o mejorar la salud; esto se logra a través del conjunto o la suma de muchas estructuras emocionales de los integrantes del grupo, de las actividades expuestas, de los silencios, conductas, actividades, diálogos, en donde el grupo va modificando su comportamiento y a través de dichas transformaciones paulatinas, sus integrantes pueden lograr una estabilidad emocional.

El grupo como dispositivo que contribuye a elevar la calidad de vida de quienes lo integran es auténtico y genuino $y$, por lo mismo, se recomienda dirigir el aprendizaje en cualquier tipo de población en forma grupal. Dentro de la población de adultos mayores existen cambios importantes como: la jubilación, el alejamiento de los hijos, las muertes de compañeros, de parejas, de amigos, de la autoimagen y otras pér- 
didas más. Al formar un grupo donde los integrantes tengan características similares les da sentido de pertenencia, ya que actúa como sostén, apoyo y como pilar afectivo (Fundación Cáritas Bienestar del Adulto Mayor/ FUNBAM, 2007; Albrecht, Terrance, Johnson \& Walther, 1993; González \& Padilla, 2006; Hombrados, García \& Martimportugués, 2004; Mora, Villalobos, Araya \& Ozols, 2004).

Es necesario resaltar que los participantes de los grupos se perciban en una situación en la que nadie sabe más que ellos las dificultades por las que están pasando, de esta manera se reúnen con el propósito de ampliar sus propios conocimientos, de aprender de las experiencias de los demás de la misma manera en que consideran que a través de ellas, pueden aprender unos de otros. Además, con el propósito de mejorar su calidad de vida en dimensiones físicas, emocionales y espirituales (Bautista, 2004).

Estos grupos pueden ser coordinados por personas que no necesariamente comparten una condición, síntoma o experiencia igual a los demás miembros, aunque deben ser conocedores de las características del proceso de envejecimiento y contar con la experiencia suficiente para el manejo de grupos. El coordinador, cuando sea necesario, puede dar su punto de vista desde su perspectiva, sin aconsejar ni enjuiciar a los participantes (Bautista, 2004).

Mendoza y López (1999), y González (1994), señalan que los grupos de autoayuda son como un foro en donde las personas que están viviendo una situación similar al del otro pueden ventilar entre ellos mismos sus emociones y sentimientos, comparten sus experiencias y entre ellos mismos se ofrecen apoyo. Además este tipo de grupo de apoyo o autoayuda tiene como objetivo fundamental ofrecer a los participantes el reconocimiento y solución de un problema común, encontrar e intercambiar apoyos, poder procesar algún tipo de duelo, reto o enfermedad, lo que permitirá rescatar las capacidades de las personas para buscar soluciones en la propia comunidad y generar sus propios servicios.

Autores como Villalba (1996) y Mendoza y López (1999), sostienen que una forma de trabajo en los grupos, transita desde el conocimiento de los miembros al desarrollo de grupo como tal, para finalmente lograr la autonomía del mismo.
El artículo describe el trabajo efectuado con un grupo pequeño de adultos mayores con el objetivo de influir en su calidad de vida, ya que se ha visto que ésta puede mejorar en dimensiones tales como: salud mental, apoyo social, autonomía y actividad físico-recreativa a través de dispositivos grupales que permitan la autogestión, así como proponer se sigan formando este tipo de grupos que ayudarán a la sociedad a promover un envejecimiento saludable, digno y exitoso.

\section{MÉTODO}

Se llevó a cabo un estudio cualitativo cuyo propósito fue formar un grupo de ayuda, creando a la vez un espacio de escucha y de diálogo para las participantes. Se elaboró un programa para ser llevado a cabo en ocho sesiones, una a la semana, con una duración promedio de 90 minutos, dicho programa estuvo abierto a los cambios sugeridos por los participantes; a fin de generar un grupo que, una vez finalizado el programa de intervención, fuera gestivo, además de ser grupo de ayuda y de crecimiento.

\section{Participantes}

En el presente estudio participaron ocho personas adultas mayores, todas mujeres, en forma voluntaria y que asistían al Centro de Salud de Banderilla Veracruz, México; con edades que oscilaban entre los 61 a 72 años, la mayoría analfabetas. Los criterios de inclusión fueron tener disposición de tiempo, no contar con alguna discapacidad mental, auditiva, visual o motriz, poder y desear asistir a las sesiones, así como ser mayores de 60 años de edad. El muestreo fue no probabilístico por conveniencia.

\section{Escenario}

Se utilizó un salón de usos múltiples del Centro de Salud mencionado, con buena iluminación y sin estímulos externos que pudieran perjudicar la atención de las personas mayores como ruidos o interrupciones ajenas; además contaba con materiales como sillas y grabadora.

\section{Instrumento}

Se elaboró una guía de entrevista con preguntas abiertas (para opiniones y creencias) y cerradas (datos sociodemográficos). Las preguntas abiertas hacían énfasis 
en cuatro categorías principales: salud general, soporte social, autonomía y actividades físico recreativas, cada una de estas categorías tenían de tres a cinco preguntas abiertas y pertinentes a la cuestión a tratar. Esta guía de entrevista fue llevada a cabo individualmente durante la primera sesión y las respuestas fueron grabadas y transcritas de manera textual, tomándose las partes más relevantes para la investigación, ya que las mujeres solían explayarse ampliamente en algunas cuestiones.

\section{Procedimiento}

Primera fase: invitación dentro del Centro de Salud a las personas mayores que estuvieran interesadas en formar el grupo de ayuda.

Segunda fase: entrevistas individuales a las ocho integrantes del grupo de ayuda, basándose en la guía de entrevista.

Tercera fase: revisión de la información obtenida de las entrevistas iníciales, y detección de las necesidades más frecuentemente reportadas por las adultas mayores en conjunto, las cuales sirvieron de base para ser tratadas en el grupo de ayuda. Inicio oficial de las sesiones. En cada sesión se hizo una retroalimentación a través de preguntas de reflexión para ver qué resultado obtuvo el tema manejado, con la finalidad de que las participantes pudieran expresar sus inquietudes y mejorar el grupo de ayuda.

Cuarta fase: la intervención concluyó en la octava sesión, donde se evaluó tanto a la coordinadora como al grupo de ayuda con las opiniones de las personas mayores acerca de la manera en que se vio beneficiada su vida en general.

A partir de los resultados obtenidos en la entrevista inicial se trabajaron los siguientes temas con la dinámica establecida y sus posibles aplicaciones: autoestima, proyecto de vida, redes sociales, autonomía, cuidado de la salud, uso del tiempo libre. Una vez que se explicó a las participantes en qué consiste un grupo de ayuda, se dio inicio al programa de intervención. La coordinadora lanzaba el tema al aire, hablando brevemente del mismo, y las mujeres comentaban sus experiencias al respecto, después otra u otras se identificaban con el problema, una de las normas establecidas fue que no se dieran consejos ni se juzgara, lo que sí podía hacerse era mencionar qué habían hecho, si era el caso, para resolver su problema y darle ánimos a la compañera para seguir adelante. La coordinadora cerraba la sesión emitiendo su opinión (sin juzgar ni aconsejar) y preguntando a las integrantes del grupo qué aprendieron, cómo se sintieron y cómo podrían aplicar lo que aprendieron en su vida diaria. Cuando surgía algún tema emergente, o sea, no planeado, era tratado con la misma importancia y del mismo modo que los demás.

En cada sesión una de las participantes era designada para dar la voz a quien deseaba participar para que se conservara el orden. Sólo intervenía aquella que deseaba hacerlo, no se presionó a ninguna para que hablara. Para cada sesión la coordinadora iniciaba el tema y las participantes llevaban el manejo de la sesión; entre todas trataban de encontrar posibles soluciones a la problemática planteada.

\section{RESULTADOS}

En la entrevista inicial se obtuvo la siguiente información: seis de las participantes padecen diabetes, y una, además, hipertensión arterial; seis no han tenido ninguna hospitalización a lo largo de su vida; siete no reportan ninguna discapacidad y una informa disminución de sus capacidades visuales; ninguna reporta adicciones a drogas, tabaco o alcohol. En cuanto al cuidado de su salud, seis de ellas acuden periódicamente al médico para el control de su enfermedad (diabetes), una también realiza ejercicio y dos no hacen nada en especial para conservar o cuidar su salud. Finalmente, con relación a cómo se autoperciben, cuatro se sienten cansadas y desmotivadas; una, vieja, inservible, cansada y desmotivada; otra, sola, cansada e inservible y dos de ellas están contentas y satisfechas con todo lo que han realizado.

En el rubro soporte social se incluyó número y parentesco con habitantes de la casa, apoyo emocional y económico, amigos y confidentes fuera de la casa, grupos de pertenencia y relaciones interpersonales.

Todas reciben apoyo económico del programa Oportunidades del gobierno federal. En cuanto al apoyo emocional, seis se sienten apoyadas por algún familiar, una se refugia en su religión y otra no recibe apoyo emocional de nadie. Ninguna de ellas tiene amigos o confidentes en los que se apoye fuera de casa, ni grupos de pertenencia o referencia dónde puedan hablar de lo que les preocupa emocionalmente, o bien, 
de su situación propia de la edad. En este mismo tenor, todas ellas opinaron no tener ningún tipo de relación con las personas de dentro (en su mismo techo) y fuera de su casa.

En el aspecto de autonomía se engloba la autonomía física y emocional y la independencia económica. Todas se sienten independientes en cuanto a que todavía son capaces de moverse por sí mismas y hacer sus quehaceres cotidianos; sólo dos de ellas se sienten independientes económicamente, ya que se sostienen de vender comida, las demás se sienten dependientes de sus familiares, aún cuando cuentan con los recursos que les proporciona el programa Oportunidades, ya que consideran que es muy escaso. En relación a afrontar sus problemas de tipo emocional, casi todas lo hacen solas, sin la ayuda ni el apoyo de nadie externo, sino por ellas mismas.

Las actividades físico-recreativas abarcan las actividades de ocio, el proyecto de vida y sus motivaciones. Todas consideraron no tener actividades de ocio. Ninguna tiene un proyecto de vida ni sabe qué quiere para su futuro ni lo ha pensado y sus principales motivaciones están en: cuidar de su nieto (1), cuidar sus animales y plantas (2), hacer ejercicio (1), vender comida (2), y no tiene ninguna motivación en la vida (2).

En general puede decirse que estas personas mayores reportaron al inicio del programa: falta de motivación que abarca desde no realizar actividades que mejoren su calidad de vida hasta no tener metas a corto plazo. En cuanto a sus relaciones sociales la mayoría vive con los hijos; sin embargo, algunas se valen por sí mismas económica y emocionalmente; la mayoría no establece relaciones de amistad con otras personas por lo que sólo se apoyan en la familia si cuentan con ella y en otros casos en ellas mismas. No asistían a ningún grupo salvo algún tipo de reunión religiosa. Su salud física se encuentra estable, no obstante pertenecen a la población diabética e hipertensa que atiende el Centro de Salud, aunque la mayoría no tiene adherencia al tratamiento debido a que no cuentan con los recursos económicos para llevar a cabo el plan de acción.

Basándose en los resultados de las entrevistas iníciales se dio paso a integrar el grupo de ayuda, que en cada sesión retomó cada uno de estos puntos débiles descritos.
Durante la octava y última sesión se realizó la evaluación del programa por parte de las participantes, con las siguientes preguntas:

¿Qué beneficios les trajo el grupo de ayuda? "Me ayudó a pensar en mí misma", "me dediqué un día a la semana para asistir al grupo", "hice nuevas amistades", "encontré una motivación para seguir viviendo", "me sirvió para desahogarme y decir cosas que nunca había dicho", "dije muchas cosas por las que pasé y que no había contado", "aquí hay gente que tiene tiempo para escucharme", "ahora que vengo a este grupo ya me siento bien, me distraigo y puedo contar algunos de mis problemas que vengo cargando desde hace mucho tiempo", "en algunas ocasiones he desahogado mis penas en este grupo", "me di cuenta que a veces es bueno decir lo que nos pasa, además, me gusta venir al grupo para distraerme y es un tiempo que yo me doy", "este grupo me trajo mucha tranquilidad y distracción, además de que encontré compañeras que pasan por mi misma situación", "voy a formar un grupo de ejercicios en mi comunidad, al fin que todos se paran temprano a caminar por los alrededores", "yo siento que ahora sí dedico tiempo para mí y cuido mi salud", "yo todos los días vengo a hacer ejercicio aquí en el centro de salud y cuando no puedo venir salgo a caminar o si no los hago en mi casa antes de dormir", "ahora ya cuido mi alimentación porque soy diabética, me tomo mi medicina y trato de hacer cosas que me gusten como salir, cuidar mis plantas, hacer ejercicio y demás cosas... me ayudan a sentirme bien", "mi alimentación si me la cuido pero la verdad es muy difícil porque yo siento que uno vive con muchas personas y como va a estar haciendo comida diferente para todos, además el dinero no alcanza, se debe uno ajustar a lo que tiene... yo sé que es por mi bien pero cómo le hago".

A partir de detectar las situaciones por las que pasan determinados sectores de adultos mayores, la sociedad ha tenido que reaccionar con nuevas respuestas de participación ante las necesidades de escucha de las personas, ésta es una de las razones que explican la formación de grupos de ayuda y no sólo en los países más desarrollados, sino también en los que están en vías de desarrollo. Este movimiento comprende: el autocuidado, que son las actividades de salud que la gente lleva a cabo para ellos o para los que se encuentran a su alrededor (Roca \& Villalbí, 1989). 
Las mujeres de este grupo de ayuda se volvieron más participativas, más para sí mismas que para los otros, ya que ser para los otros es un papel que la sociedad les ha enseñado a través de toda una memoria colectiva cultural. Estar en el grupo les ha servido para poner más atención y dedicarse más al cuidado de su salud, y, sobre todo, para visibilizarse comos mujeres, como sujetos potenciales del desarrollo, para lograr superar las visiones fragmentadas que las consideran "grupos vulnerables" o ciudadanas de segunda categoría.

El grupo de ayuda intenta restablecer el orden, lo cual implica el compromiso por desarrollarse intencionalmente y aprender junto con otras mujeres con el mismo fin, con el objetivo de aumentar, de ensanchar su poderío de género desarrollando su capacidad para tomar decisiones sobre eventos importantes en sus vidas, incrementar recursos, oportunidades para ejercer sus derechos y poderes civiles, políticos y culturales.

\section{¿Qué les gustó y qué no les gustó?}

En consenso declararon: "nos gustó todo del grupo de ayuda, la forma en que lo dirigimos y de lo que hablamos, además de las actividades que en cada sesión hicimos", "nos ayudó a pensar en nosotras mismas y dedicarnos un día a la semana para asistir al grupo", "aquí hicimos nuevas amistades y encontramos un motivo para seguir viviendo".

De acuerdo a Sánchez (2002) la estructura de un grupo es como el patrón de sus relaciones que surge entre sus miembros, además de que modera las tensiones entre los miembros regulando y controlando las relaciones. De esta manera la estructura de un grupo es elemental, consolidada de tal manera que, cuando se desarrolla, muy difícilmente puede cambiarse, trayendo beneficios como lo es mayor fluidez, mejor comunicación entre los miembros y, por ende, proporciona mejores resultados.

Sentirse parte de un grupo proporcionó a estas mujeres una seguridad que proyectaron hacia sus hogares y hacia la comunidad; les brindó, en gran medida, un bienestar emocional al encontrarse acompañadas de otras mujeres que encaraban aún situaciones más difíciles y que, de alguna manera, las motivaban a seguir adelante, a no amedrentarse ante los problemas y a pensar que las cosas pueden cambiar y si no se cam- bian, puede transformar la forma de verlas y de enfrentarse a ellas.

\section{¿Cómo influyó este grupo en su vida?}

"Había días en que me sentía muy sola porque mis hijos ya casi no vienen a verme, pero desde que me metí al grupo poco a poco se me fueron quitando esas ideas de tristeza; dejé de pensar en que estaba sola y ahora si mis hijos no me van a ver ya no me preocupo, se que yo sola puedo valerme por mí misma, si quiero me hago de comer, salgo, voy al grupo, si no quiero no hago de comer y nadie me dice nada"; "aunque vivo sola mis nietos están conmigo, ya que los cuido mientras su mamá trabaja; ellos me han ayudado mucho a no sentirme sola"; "hace un tiempo me sentía muy sola y todo el día no hacía otra cosa más que llorar y llorar, ahora vivo sola y aunque no tengo mucho dinero como lo que hay, si no tengo nada me corto unos quelites y con frijolitos me los como, riego mis plantitas, cuido mis animalitos o siembro plantitas o quelites y así me ayudo para comer, me siento muy contenta y tranquila de ya no estar triste como antes..."; "desde los nueve años tuve que trabajar como sirvienta para poder comer un plato, además de que cuidaba de mis hermanos...mi papá era alcohólico, le pegaba a mi mamá y ahora a mí me toca repetir la historia con mi marido, él toma mucho..."; "ustedes no se caigan por los problemas, lo que no mata engorda... siempre hay que mirar hacia delante y afrontar lo que se nos venga..."; "toda la vida me la pasé pensando en los demás y resolviendo sus problemas, ahora me preocupo por mí cuando me siento triste, que mis problemas me absorben, me encierro en mi casa, apago todo y solamente cierro los ojos y me pongo a descansar, a veces me salgo a caminar y dejo todo, me vale si hay quehacer o no..."; "yo he enterrado a mis padres, mis hermanos... me ha tocado cuidarles las enfermedades porque murieron de cáncer, mi hijo a veces me chantajea que se quiere quitar la vida, toma igual que su padre... y a mí me tiene que ver fuerte por más que me duele no se lo demuestro, porque si a mí me ven mal la familia se cae"; "mi esposo siempre tomó mucho, me golpeaba y me daba muy mala vida, hasta que murió por lo mismo. Ahora yo vivo sola, mis hijos viven cerca de mi casa y cuando necesito algo ellos me dan y me atienden, me siento muy contenta y tranquila"; "sí, yo he pasado por cosas 
muy fuertes, pero aquí sigo dando lata, por eso les digo que no hay nada que nosotras las mujeres no podamos enfrentar, yo sólo me encomiendo a Dios y él será el que decida llevarme, pero mientras yo sigo adelante".

A la fecha aún no se ha llegado a un consenso sobre la definición de apoyo social, ya que se refiere a un complejo fenómeno, compuesto por varias dimensiones, que se asocia con la salud de los individuos. Sin embargo, la investigación sobre el apoyo social sostiene que la presencia o ausencia de éste afecta diferencialmente la salud de los individuos. La naturaleza de esta relación explicaría porqué los individuos con relaciones de apoyo (en forma de familia, amigos, pareja, entre otros), con frecuencia tienen mejores condiciones de salud física y mental - dados los recursos emocionales y/o materiales que obtienen de estas relaciones(Castro, Campero \& Hernández, 1997).

Algunos autores consideran que la forma más efectiva de apoyo depende de la situación en que éste es necesario (Matud, Carballeira, López, Marrero \& Ibáñez, 2002).

Este grupo de ayuda manejó primordialmente el apoyo emocional; se trataba de que se dieran cuenta de que se encontraban en situaciones similares y que ellas mismas podían encontrar la solución, si bien no era la intención aconsejar, si lo era exponer ideas y lograr un aprendizaje significativo, un aprendizaje para la vida a través del apoyo social.

¿Cuál es la idea que tienen ahora de ser una mujer mayor? "Es una parte de la vida difícil"; "es más difícil cuando no se tiene a nadie"; "aunque sea difícil ya estar grande se tiene que disfrutar y sacarle provecho a lo que se tiene"; "debemos pensar en nosotras mismas"; "debemos aprender a no necesitar tanto de los demás para poder resolver nuestros problemas"; "que aunque ya esté vieja hay mucho todavía que vivir y disfrutar".

El autoconcepto juega un papel decisivo y central en el desarrollo de la personalidad, tal como lo destacan las principales teorías psicológicas, un autoconcepto positivo está en la base del buen funcionamiento personal, social y, en su caso, profesional, dependiendo de él, en buena medida, la satisfacción personal, el sentirse bien consigo mismo (Esnaola, Goñi \& Madariaga, 2008). De ahí que el logro de un equilibrio socioafectivo en las personas mayores a partir de una imagen ajustada y positiva de sí mismas figure entre las finalidades del programa de ayuda que se llevó a cabo con ellas. De ahí también que lograr un autoconcepto positivo sea uno de los objetivos pretendidos en los grupos de ayuda con intervención psicológica reeducativa, comunitaria y cívica, y para los que se demandan estrategias y recursos que permitan elevar la calidad de vida de quienes participan en ellos.

\section{DISCUSIÓN}

Al comparar los resultados encontrados en la entrevista inicial en el grupo de mujeres participante y haciendo referencia a las categorías estudiadas, que son: estado de salud en general, soporte social, autonomía y actividades físico recreativas, se resume lo siguiente:

Al realizar la evaluación del grupo de ayuda, o sea, al finalizar las sesiones programadas, las opiniones de las personas con respecto a ellas mismas y su manera de ver y vivir la vida dio un giro de manera positiva; las personas mayores reportaron sentirse a gusto con su etapa de vida, creando la autogestión personal y metas a corto plazo, sus relaciones sociales se vieron beneficiadas encontrando en sus compañeras disposición para la escucha activa, aceptación, solidaridad, apoyo emocional y compañerismo, además de tomar al grupo de ayuda como un momento para ellas mismas. $\mathrm{Su}$ salud física también se vio beneficiada, ya que adoptaron hábitos saludables como realizar ejercicio por las mañanas además de llevar a cabo el tratamiento para la diabetes e hipertensión. De esta manera se cumple con la definición de calidad de vida de Katschnig (2000), quien menciona que ésta es un conjunto de factores que tienen que ver con la salud, afrontamiento, solución de problemas, control, auto-eficacia y el desarrollo de habilidades sociales, tanto en la familia, como en la escuela, trabajo, o dentro de una comunidad.

Inga \& Vara (2002) realizaron una investigación sobre calidad de vida en proyectos donde hacen referencia a ciertos estilos de vida dañinos que disminuyen la probabilidad de tener una calidad de vida exitosa en los adultos mayores; casualmente las participantes del grupo de ayuda presentaban todos esos estilos nocivos mencionados por estos autores, lo cual hacía que su calidad de vida fuera precaria. Dentro del grupo de ayuda pudieron solucionarse en gran parte, ya que lograron crear un estilo de vida adecuado para su salud, 
obtuvieron un aprendizaje significativo sobre su etapa de vida, lograron encontrar actividades motivantes y felicitantes para su vida diaria, y, por último, pudieron relacionarse socialmente encontrando un lazo emocional con sus compañeras. Todo lo cual apoya la tesis de que el grupo de ayuda es benéfico en cualquier tipo de población, ya que ayuda a equilibrar de manera drástica el estado emocional beneficiando la calidad de vida, en especial, de las personas mayores.

Es recomendable para los profesionales de la salud en el campo de la psicología que tomen en cuenta este tipo de programas y sobre todo a la población de adultos mayores. El grupo de ayuda es una herramienta de gran importancia porque se puede lograr un cambio positivo en las personas que participan en él, al integrarlos y lograr una cohesión del grupo puede mejorar la situación emocional, si es el caso, que presentan las personas de este grupo etario.

Los grupos de ayuda, como lo menciona Bautista (2004) son organizaciones de personas que comparten problemas psicológicos, físicos o existenciales similares; donde los participantes se reúnen con el propósito

\section{REFERENCIAS}

Albrecht, T., Johnson, G. \& Walther, J. (1993). Understanding communication processes in focus groups. Newbury Park: Sage Publications.

Bautista, A. (2004). La comunicación nos libera. Los grupos de ayuda como espacios de encuentro. Cali: Magisterio.

Carretero, M. Palacios, J. \& Marchesi, A. (2000). Psicología evolutiva: Adolescencia, madurez y senectud. Madrid: España.

Castro, R., Campero, L. \& Hernández, B. (1997). La investigación sobre apoyo social en salud: situación actual y nuevos desafíos. Revista de Saúde Pública, 31(4), 425-435.

Esnaola, I., Goñi, A. \& Madariaga, J. (2008). El autoconcepto: perspectivas de investigación. Revista de Psicodidáctica, 13(1), 179-194.

Fundación Cáritas Bienestar del Adulto Mayor (FUNBAM) (2007). Resultados del proyecto: Sistematización del proceso de conformación, funcionamiento y fortale- de ampliar sus propios conocimientos, aprender de las experiencias de los demás de la misma manera en que consideran que a través de ellas pueden aprender unos de otros, y/o desean mejorar su calidad de vida. Dentro de esta investigación se pudo constatar el concepto anterior, ya que las mismas participantes expusieron las principales situaciones que les aquejaban, encontrando en ellas mismas un apoyo emocional, lográndose una adaptación grupal, compañerismo, solidaridad, responsabilidad y disposición.

En relación al tipo de población con la que se trabajó, con sus grandes desventajas de género, de pobreza, de exclusión social y familiar, y, sobre todo, de edad, hoy en día poco o nulamente es tomada en cuenta para realizar un programa en beneficio a su salud. Se establecen pocos programas de salud porque la mayor parte del presupuesto público está destinado a la niñez y a las mujeres en edad reproductiva; es por lo anterior que se sugiere este tipo de intervenciones que no necesitan de gran presupuesto y contribuyen a que el adulto mayor se sienta útil y valorado por la sociedad a la que pertenece.

cimiento de redes de apoyo de adultos mayores originadas por la operación del Programa de Educación no Formal, en el Distrito Federal y el estado de México, en Red latinoamericana de Gerontología. Recuperado el 9 de noviembre de 2009 de: http://www.gerontologia.org/portal/archivosUpload/InestigacionFunbamRedes2006.pdf

García, M. (2003). Trabajo social en gerontología. México: Síntesis.

González, A. (2000). Cuando la tercera edad nos alcanza. México: Trillas.

González, A. \& Padilla, A. (2006). Calidad de vida y estrategias de afrontamiento ante problemas y enfermedades en ancianos de Ciudad de México. Facultad de Estudios Superiores Iztacala, UNAM. Recuperado el 17 de enero de 2010 de: http://sparta.javeriana.edu. co/psicologia/publicaciones/actualizarrevista/archivos/V5N304_calidadvidaestrategiasafrontamiento. pdf 
González, J. (1994). Dinámica de grupos, técnicas y tácticas. México: PAX México.

Gutiérrez, F. \& Prado, C. (2000). Género y Nueva ruralidad. San José de Costa Rica: Instituto Americano de Cooperación para la Agricultura (IICA) y Centro Internacional de Desarrollo Rural (CIDER).

Hombrados, M. García, M. \& Martimportugués, C. (2004). Social support groups for the elderly: a methodological proposal for development and assessment. Universidad de Málaga. Recuperado el 17 de enero de 2010 de: http://dialnet.unirioja.es/servlet/articulo ?codigo $=1004533$

INEGI (2006). Censo de Población y vivienda 2005. Recuperado el 7 de agosto de 2010 de http://www.inegi. gob.mx/inegi/default.aspx

Inga, A. \& Vara, H. (2002). Calidad y satisfacción de vida en adultos mayores de 60 años en Lima, Perú. Recuperado el 12 de enero de 2010 de http:www. geocities.com/Heartland/5900/adulto02.htm

Katschnig, H. (2000). Utilidad del concepto de calidad de vida en psiquiatría. Barcelona: Masson.

Marín, M. \& Garrido, T. (2003). El grupo desde la perspectiva psicosocial. Conceptos Básicos. Madrid: Pirámide.

Matud, P., Carballeira, M., López, M., Marrera, R. \& Ibañez, I. (2002). Apoyo social y salud: un análisis de género. Salud Mental, 25(2), 32-37.
Mendoza, L. \& López, E. (1999). Manual de grupos de autoayuda. Sugerencias para organizar, establecer $y$ dirigir grupos de autoayuda. Recuperado el 18 de noviembre de 2009 de http://www.sideso.df.gob.mx/ documentos/manual_de_grupos_de_autoayuda.pdf

Mora, M., Villalobos, D., Araya G. \& Ozols A. (2004). Perspectiva subjetiva de la calidad de vida del adulto mayor, diferencias ligadas al género y a la práctica de la actividad físico recreativa. Revista MH Salud, 1(1) Recuperado el 09 de noviembre de 2009 de http://www.una.ac.cr/mhsalud/documents/ ARTICULOMONICAMORA_017.pdf

Roca, F. \& Villalbí, J. (1989). Los grupos de ayuda mutua en el campo de la salud. Revista de Trabajo Social, $114,45-51$.

Sánchez, J. (2002). Psicología de los grupos: Teorías, procesos y aplicaciones. Barcelona: McGraw-Hill.

Triado, C. \& Villar, F. (2006). Psicología de la vejez. Madrid: Alianza.

Villalba, C. (1996). Los grupos de apoyo basados en la autoayuda: una propuesta para el inicio y acompañamiento profesional. Intervención Psicosocial, 15, 23-41. 